\title{
Qualité hygiénique du fromage de "fosse" de Sogliano al Rubicone (Italie)
}

\author{
S. MASSA *(1), G.C. TURTURA *, L.D. TROVATELLI ** \\ * Università di Bologna, Istituto di Microbiologia Agraria e Tecnica, Via Filippo Re 6, \\ 40126 Bologna, Italy \\ ** Università della Tuscia, Istituto di Microbiologia e Tecnologie Agrarie, Loc. Riello, \\ 01100 Viterbo, Italy
}

\begin{abstract}
Résumé
Des analyses microbiologiques ont été réalisées sur 30 échantillons de fromage de «fosse ", produit typique de la région de Sogliano al Rubicone (Italie), afin d'en évaluer la qualité hygiénique. Aucun échantillon ne contenait de coliformes ou de salmonelles. La flore totale et les entérocoques oscillaient respectivement d'un minimum de $6,5 \cdot 10^{4}$ et $9,5 \cdot 10^{3}$ à un maximum de $2,2 \cdot 10^{6}$ et $8,1 \cdot 10^{4}$ à g. Bacillus cereus et Clostridium perfringens furent trouvés en nombre très réduit uniquement dans quelques échantillons, tandis que dans $10 \%$ de ceux-ci Staphylococcus aureus variait de $7 \cdot 10^{2}$ à $4 \cdot 10^{3}$ par g. Parmi les entérocoques les espèces les plus fréquentes furent Streptococcus faecium $(40 \%)$, Str. faecalis var. liquefaciens (35\%), Str. faecalis var. faecalis (22\%) et Str. durans $(3 \%)$.

Mots clés: Fromage de "fosse" - Qualité hygiénique - Enterococci - S. aureus $B$. cereus - $\mathrm{Cl}$. perfringens.
\end{abstract}

\section{Summary}

Hygienic quality of Sogliano "pit " cheese (Italy)

A microbiological analysis for hygienic evaluation was performed on 30 samples of Sogliano al Rubicone «pit» cheese. Neither coliforms nor salmonella were found. Total aerobic flora and enterococci ranged from $6.5 \times 10^{4}$ and $9.5 \times 10^{3}$ to $2.2 \times 10^{6}$ and $8.1 \times 10^{4} / \mathrm{g}$, respectively. Bacillus cereus and Clostridium perfringens were found in very small numbers in very few samples. In $10 \%$ of the samples Staphylococcus aureus varied from $7 \times 10^{2}$ to $4 \times 10^{3} / \mathrm{g}$. The most frequent species of enterococci were Str. faecium $(40 \%)$, Str. faecalis var. liquefaciens $(35 \%)$, Str. faecalis var. faecalis (22\%) and Str. durans $(3 \%)$.

Key words : «Pit» cheese - Hygienic quality - Enterococci - S. aureus - B. cereus Cl. perfringens.

(1) Auteur qui recevra la correspondance 


\section{Introduction}

Le fromage de «fosse » est un fromage local typique, ainsi appelé car la fermentation se produit pendant une période de trois mois dans des trous ou "fosses » creusés dans le tuf (roche tendre) de la zone de Sogliano al Rubicone (Italie). Il s'agit d'un fromage à pâte dure, produit avec du lait entier et non pasteurisé de vache, de brebis ou de mélange des deux espèces. Sa technique de fabrication a été décrite dans une étude précédente (MASSA et al., 1986).

Les connaissances microbiologiques et physicochimiques du fromage de «fosse » sont encore fragmentaires. L'unique étude de la microflore responsable de la fermentation a révélé qu'un nombre relativement élevé de bactéries lactiques (MASSA et al., 1986) persiste en fin de maturation. Des entérocoques sont également présents tandis que les coliformes et les clostridium sont absents (moins de $10 \mathrm{ufc} / \mathrm{g}$ ). Lorsque les fromages sont prélevés dans les fosses où ils fermentent à une température d'environ $21^{\circ} \mathrm{C}$, ils présentent un $\mathrm{pH}$ qui oscille de 4,90 à 5,20 et une humidité de 27,6 à $31 \%$ suivant le type de fromage (de vache, de brebis ou mixte).

Mis à part le travail de Massa et at. (1986), qui comporte une analyse partielle de la flore d'intérêt hygiénique (coliformes et streptocoques fécaux), aucune donnée concernant la qualité hygiénique du fromage de «fosse » n'est disponible dans la littérature.

Des toxiinfections alimentaires, bien que relativement rares d'un point de vue épidémiologique, ont été associées à la consommation de fromages fabriqués avec du lait cru ou pasteurisé (MARTH, 1969 ; Minor et MARTH, 1972 ; Marier et al., 1973 ; Fontaine et al., 1980 ; Ricci et al., 1984 ; RATnAm et MARCH, 1986).

Le but de cette étude a été d'apprécier la qualité hygiénique du fromage de «fosse » en procédant à la numération des groupes microbiens ayant une signification en la matière ; on a voulu aussi vérifier la présence de certains germes d'importance particulière pouvant être à l'origine de toxiinfections alimentaires. Parmi les microorganismes pathogènes n'ont pas été considérés Mycobacterium tuberculosis et Brucella spp., puisque le lait provenait d'élevages officiellement indemnes de tuberculose et brucellose.

\section{Matériel et méthodes}

\section{A. Echantillonnage et préparation des échantillons}

Les échantillons étudiés provenaient de six lots fabriqués à partir de lait cru selons des procédés artisanaux dans six usines différentes de la zone de Sogliano al Rubicone. 30 échantillons - cinq à lots - ont été prélevés dès l'ouverture des fosses ; transportés au laboratoire, sous la forme de portions entières de fromage de poids variant de 0,5 à $1,3 \mathrm{~kg}$, ils furent examinés dans les 24 heures. 
Pour l'examen microbiologique une part globale de $50 \mathrm{~g}$ a été utilisée, après avoir éliminé la couche externe avec les techniques habituelles d'asepsie. Après dilution à $1: 10$ dans une solution de citrate de sodium à $2 \%$ (MARSHALL et al., 1985) on a procédé à l'homogénéisation de l'échantillon à l'aide d'un Stomacher 400 Colwort (Thrasher et Richardson, 1980). Les dilutions décimales successives furent ensemencées sur les différents substrats en employant $0,1 \mathrm{ml}$ par étalement ou $1 \mathrm{ml}$ par incorporation.

Le $\mathrm{pH}$ de chaque échantillon fut déterminé au moyen d'un $\mathrm{pH}$ mètre Beckman sur une suspension de fromage.

\section{B. Numération des microorganismes}

Outre les différents groupes microbiens mentionnés au tableau 1, ont été dénombrés les staphylocoques dorés et les salmonelles; ces dernières ont été recherchées qualitativement dans $25 \mathrm{~g}$ de chaque échantillon.

Pour Staphylococcus aureus le milieu Baird Parker Base Agar (Biolife) ensemencé par étalement a été utilisé. Après $24 \mathrm{~h}$ à $37^{\circ} \mathrm{C}$ fut compté le nombre $(\mathrm{n})$ de colonies noires typiques. Une partie de celles-ci $(\sqrt{n})$ furent soumises aux tests de la catalase, de la DNase et de la coagulase et à un examen microscopique.

Pour les salmonelles on a procédé à un préenrichissement en eau peptonée tamponnée, avec incubation à $37^{\circ} \mathrm{C}$ pendant $24 \mathrm{~h}$, puis à un enrichissement sur deux substrats simultanément: Selenite Cystine Broth (Biolife) et Tetrathionate Broth Base (Biolife), incubés respectivement à $37^{\circ} \mathrm{C}$ et $44^{\circ} \mathrm{C}$ pendant 24 et $48 \mathrm{~h}$; l'isolement était effectué sur les substrats sélectifs suivants : SS Agar (Biolife), Bismuth Sulphite Agar (Biolife) et Brillant Green Agar (Biolife), tous incubés à $37^{\circ} \mathrm{C}$ pendant 24 à $48 \mathrm{~h}$.

\section{Identification}

Pour les entérocoques ont été étudiés les caractères d'identification suivants : morphologie, production de catalase, croissance sur Aesculin Bile Agar (Biolife) et Brain Heart Infusion (Biolife) contenant 6,5\% de $\mathrm{NaCl}$, croissance sur milieu Tryptose Blood Agar Base (Oxoid) au tellurite de potassium (concentration finale de $0,05 \%$ ), résistance à $60^{\circ} \mathrm{C}$ pendant $30 \mathrm{mn}$ sur milieu de base BHI, hémolyse sur Tryptic Soy Agar (Biolife) additionné de $5 \%$ de sang de mouton, hydrolyse de la gélatine d'après la technique décrite par Frasier (TIECCO, 1984). Des tests biochimiques complémentaires ont été faits en utilisant le système API 20 STREP.

La présence de $B$. cereus et $C l$. perfringens a été confirmée en se basant sur les caractères biochimiques et physiologiques décrits par HARMON et GoEPFERT (1984) et par HARMON et DUNCAN (1984). 
Tableau 1

Numération des microorganismes du fromage de «fosse ». Milieux utilisés et conditions d'incubation Enumeration of microorganisms in "pit» cheese. Media and incubation conditions

\begin{tabular}{|c|c|c|c|c|}
\hline $\begin{array}{l}\text { Groupe de } \\
\text { microorganismes }\end{array}$ & $\begin{array}{l}\text { Milieux } \\
\text { utilisés }\end{array}$ & \multicolumn{2}{|c|}{ Incubation } & Observations \\
\hline Flore totale & $\begin{array}{l}\text { Plate Count Agar (PCA) } \\
\text { (OXOID) }\end{array}$ & 32 & 72 & $\begin{array}{l}\text { Ensemencement } \\
\text { par incorporation }\end{array}$ \\
\hline Coliformes totaux & $\begin{array}{l}\text { Violet Red Bile Agar } \\
\text { (OXOID) }\end{array}$ & 37 & 24 & $\begin{array}{l}\text { Ensemencement } \\
\text { par incorporation } \\
\text { en double couche }\end{array}$ \\
\hline Entérocoques & $\begin{array}{l}\text { Barnes Basal Medium } \\
\text { (Biolife) }\end{array}$ & 45 & 24 & $\begin{array}{l}\text { Ensemencement } \\
\text { par étalement }\end{array}$ \\
\hline Bacillus cereus & $\begin{array}{l}\text { Cereus Selective Agar } \\
\text { Base (Merck) }\end{array}$ & 32 & 24 & $\begin{array}{l}\text { Ensemencement } \\
\text { par étalement }\end{array}$ \\
\hline Clostridium perfringens & $\begin{array}{l}\text { Sulphite Polymixin } \\
\text { Sulphadiazine Agar } \\
\text { (Biolife) }\end{array}$ & 37 & 24 & $\begin{array}{l}\text { Ensemencement } \\
\text { par incorporation et } \\
\text { incubation en anaérobiose } \\
\text { dans jarre Gas Pack } \\
\text { (BBL) }\end{array}$ \\
\hline
\end{tabular}




\section{Résultats}

Les résultats relatifs aux divers microorganismes d'intérêt hygiénique sont présentés dans le tableau 2.

- $p H$ : les limites de variation du $\mathrm{pH}$ (non reporté sur le tableau) furent de 4,92 et 5,45 .

- Germes totaux : leur nombre oscillait entre $6,5 \cdot 10^{4}$ et $9,8 \cdot 10^{4}$ ufc/g dans 8 échantillons $(26,7 \%)$, tandis que les autres $(73,3 \%)$ présentaient des valeurs supérieures à $10^{5} \mathrm{ufc} / \mathrm{g}$ avec un maximum de $2,2 \cdot 10^{6} \mathrm{ufc} / \mathrm{g}$.

- Coliformes totaux et coliformes fécaux : pour aucun des 23 échantillons examinés on n'a trouvé de colonies de coliformes sur les boîtes de Petri. Compte-tenu de la dilution du fromage, le niveau des bactéries coliformes était donc inférieur à $10 \mathrm{ufc} / \mathrm{g}$.

- Entérocoques : le nombre de ces microorganismes variait d'un minimum de $9,5 \cdot 10^{3}$ ufc/g à un maximum de $8,1 \cdot 10^{4}$ ufc/g. L'espèce la plus représentée était Str. faecium $(40 \%)$, Str. faecalis var. liquefaciens $(35 \%)$, Str. faecalis $(22 \%)$ et Str. durans $(3 \%)$.

On n'a pas trouvé d'entérocoques appartenant à l'espèce Str. faecalis var. zymogenes.

- Staphylococcus aureus : seuls 3 échantillons (10\%) étaient positifs. Dans 2 échantillons de fromage de brebis on trouvait respectivement $9 \cdot 10^{2}$ et $7 \cdot 10^{2} \mathrm{ufc} / \mathrm{g}$, tandis que dans un fromage mixte le niveau était de $4 \cdot 10^{3} \mathrm{ufc} / \mathrm{g}$; dans les échantillons restants les nombres furent toujours inférieurs à $10^{2} \mathrm{ufc} / \mathrm{g}$.

- Bacillus cereus : dans 27 échantillons les nombres de $B$. cereus étaient inférieurs à $10^{2} \mathrm{ufc} / \mathrm{g}$; dans un échantillon il était de $3 \cdot 10^{2} \mathrm{ufc} / \mathrm{g}$.

- Clostridium perfringens : la présence de $\mathrm{Cl}$. perfringens se révéla seulement dans 2 échantillons de fromage mixte $\left(2 \cdot 10^{2}\right.$ ufc/g et $4 \cdot 10^{2} \mathrm{ufc} / \mathrm{g}$.

- Salmonelles : leur recherche s'est toujours révélée négative dans $25 \mathrm{~g}$.

\section{Discussion}

Parmi les index microbiens examinés, seuls la flore totale et les entérocoques présentaient des valeurs relativement élevées qui concordent, pour l'essentiel, avec celles rapportées dans notre précédent travail (MASSA et al., 1986). Toutefois, ceci ne signifie pas que la qualité hygiénique du fromage de "fosse » soit défectueuse car, outre les bactéries contaminantes, sur le milieu PCA, on dénombre un certain nombre de bactéries lactiques.

Les entérocoques sont considérés comme indésirables par les hygiénistes de l'alimentation. Mise à part leur activité bétahémolytique attribuée uniquement à l'espèce Str. faecalis var. zymogenes (donc théoriquement pathogène), il n'a pas été encore prouvé, de la part de ces bactéries, la production d'entérotoxines et, de plus, la majorité des auteurs a échoué dans la tentative d'induire expérimentalement des troubles gastro-intestinaux ou d'autre nature (Fischer, 1958 ; Linde, 1959; Deibel et Silliker, 1963). Plus récemment 
TABleau 2

Distribution des échantillons de fromage de "fosse " suivant différentes classes de microorganismes d'intérêt hygiénique. Nombre de ufc (unités formant des colonies) par $g$

Distribution of samples of «pit» cheese containing different groups of bacteria of hygienic relevance. CFU per $g$

\begin{tabular}{|c|c|c|c|c|c|c|c|}
\hline Groupe microbien & $\begin{array}{c}\text { Nombre } \\
\text { d'échantillons }\end{array}$ & Absent & $<10^{2}$ & $10^{2}-10^{3}$ & $>10^{3}-10^{4}$ & $>10^{4}-10^{5}$ & $>10^{5}$ \\
\hline Flore totale & 30 & 0 & 0 & 0 & 0 & 26,7 & 73,3 \\
\hline Coliformes totaux & 23 & 100,0 & 0 & 0 & 0 & 0 & 0 \\
\hline Coliformes fécaux & 23 & 100,0 & 0 & 0 & 0 & 0 & 0 \\
\hline Entérocoques & 30 & 0 & 0 & 0 & 60,0 & 40,0 & 0 \\
\hline S. aureus & 30 & 0 & 90,0 & 6,7 & 3,3 & 0 & 0 \\
\hline B. cereus & 28 & 0 & 96,4 & 3,6 & 0 & 0 & 0 \\
\hline Cl. perfringens & 30 & 0 & 93,3 & 6,7 & 0 & 0 & 0 \\
\hline Salmonelles & 30 & 100,0 & 0 & 0 & 0 & 0 & 0 \\
\hline
\end{tabular}


Batisch et al., $(1982,1985)$ ont démontré l'activité entérotoxique de souches de Str. faecium et Str. faecalis var. zymogenes vis-à-vis de souris et lapins, en la rapportant aux caractères suivants : thermorésistance, activité désoxyribonucléique, hémolyse. Au contraire Сomı et al. (1986) sur des souches de la même espèce d'entérocoques, considérés pathogènes d'après la thermorésistance, l'activité désoxyribonucléique et l'hémolyse, n'ont pas réussi à mettre en évidence une quelconque propriété entérotoxique.

Puisque les entérocoques se retrouvent constamment dans de nombreux fromages italiens et qu'ils ont été utilisés comme levains à titre expérimental dans des fromages étrangers, il serait plus juste de les considérer comme des microorganismes normaux dans les produits laitiers (OTrogalli et al., 1978).

Au cours de notre recherche, l'absence de Str. faecalis var. zymogenes et au contraire la présence de Str. faecalis var. liquefaciens, qui développe une action de stimulation sur la croissance et le développement des ferments lactiques (Clark et Reinbold, 1966; Devoyod et Muller, 1969), est plutôt rassurante quant à la qualité hygiénique du fromage de «fosse ».

L'absence totale des coliformes, bien que ne pouvant constituer un indice de bonne fabrication par leur comportement imprévisible durant la fermentation des fromages (PARK et al., 1973), peut sans doute s'expliquer par le $\mathrm{pH}$ bas, la faible teneur en eau du fromage et l'action antagoniste exercée par les bactéries lactiques (DALY et al., 1970 ; BABEL, 1977 ; RASH et KosIKOWSKI, 1982).

La présence de ces derniers en quantité élevée à la fin de la fermentation (MAssa et al., 1986), peut encore expliquer l'absence de salmonelles dans $25 \mathrm{~g}$ dans tous les échantillons, le très petit nombre de $\mathrm{Cl}$. perfringens et $\mathrm{B}$. cereus associé à la contamination réduite en staphylocoques dorés.

En détail, pour $S$. aureus la Commission Internationale pour les Spécifications Microbiologiques des Aliments (ICMSF, 1974) a recommandé pour les fromages à pâte demi-dure ou dure, un plan d'échantillonnage à̀ trois classes et a suggéré les seuils limites suivants de contamination : $n=5, c=1, m=10^{3}$ ufc/g, et $M=10^{4} \mathrm{ufc} / \mathrm{g}$. Le symbole $n$ signifie le nombre total des unités qui représentent le lot, $m$ signifie le niveau de contamination au-dessous duquel on peut estimer que l'unité provient d'une fabrication satisfaisante en ce qui concerne les caractéristiques bactériologiques et hygiéniques, $M$ correspond au niveau au-dessus duquel on ne doit avoir aucun échantillon, sinon le lot doit être rejeté, $c$ représente le nombre maximum d'unités qui peut contenir un nombre de microorganismes plus grand que $m$.

Si nous avions appliqué au fromage de "fosse » les seuils proposés par l'ICMSF tous les lots auraient été acceptés. En effet dans tous les échantillons le niveau de $S$. aureus était inférieur à $10^{3} \mathrm{ufc} / \mathrm{g}$; seulement dans un échantillon on dépassait cette valeur qui, toutefois, n'excédait pas le niveau de $M$.

En considérant ces propos et l'ensemble des résultats obtenus, il nous semble pouvoir conclure que, pour le type et la quantité de microorganismes rencontrés, la qualité hygiénique du fromage de « fosse » produit dans la zone de Sogliano al Rubicone est satisfaisante. Toutefois, il ne faut pas oublier que dans les fromages vieillis, bien que la flore lactique développe une action 
antagoniste envers la plupart des microorganismes d'altération et/ou de caractère pathogène, il peut rester d'éventuelles toxines produites au début du processus de maturation (RITTER, 1975): des exemples typiques sont les toxiinfections alimentaires par $S$. aureus et par B. cereus (GOEPFERT et al., 1972).

Reçu le 12 mars 1987.

Accepté pour publication le 6 novembre 1987.

\section{Références bibliographiques}

Babel F.J., 1977. Antibiosis by lactic culture bacteria. J. Dairy Sci., 60, 815-821.

Batish V.K., Chander H., Ranganathan B., 1982. Characterization of deoxyribonuclease positive enterococci isolated from milk and milk products. J. Food Prot., 45, 348-352.

Batish V.K., Chander H., Ranganathan B., 1985. Heat resistance and other characteristics of deoxyribonuclease positive enterococci isolated from milk and milk products. J. Food. Sci., $50,834-835$.

Clark W.S., Reinbold G.W., 1966. Enterococci in young Cheddar cheese. J. Dairy Sci., 49, 1214-1218.

Comi G., D'Aubert S., Cantoni C., 1986. Gli streptococci D negli alimenti di origine animale. Ind. Alim., 25, 289-290.

Daly C., Sandine W.E., Elliker P.R., 1970. Associative growth and inhibitory properties of Streptococcus diacetylactis. J. Dairy Sci, 53, 637.

Deibel R.H., Silliker J.H., 1963. Food poisoning potential of the enterococci. J. Bacteriol., 85, $827-832$.

Devoyod J.J., Muller M., 1969. La flore microbienne du fromage de Roquefort. III. Les streptocoques lactiques et les Leuconostoc. Influence de différents microorganismes de contamination. Lait, 49, 369-399.

Fischer J., 1958. Nachveismethode für eingepflatze körperfremde Enterokokken (D. Streptokokken) und ihre klinische Brauchbarkeit. Zentralbl. Bakleriol. Parasitenk. Infectionskr. Hyg. Abt. 1: Orig. Reihe B., 171, 264-271.

Fontaine R.E., Cohen M.L., Martin W.T., Vernon T.M., 1980. Epidemic salmonellosis from Cheddar cheese. Am. J. Epidemiol., 111, 247-253.

Goepfert J.M., Spìra W.M., Kim H.W., 1972. Bacillus cereus : food poisoning organisms. A review. J. Milk Food Technol, 35, (4), 213-227.

Harmon S.M., Goepfert M.J., 1984. Bacillus cereus. In: Compendium of methods for the microbiological examination of foods, Speck M.L. Ed., Washington D.C., 464-467.

Harmon S.M., Duncan C.L., 1984. Clostridium perfringens. In : Compendium of methods for the microbiological examination of foods, Speck M.L. Ed., Washington D.C., 489-491.

ICMSF (International Commission on Microbiological Specifications for Foods), 1974. Microorganisms in foods. 2. Sampling for microbiological analysis: Principles and specific applications. University of Toronto Press, Toronto, Ontario, Canada.

LINDE K., 1959. Über enteropathogene Darmstreptokokken. Zentralbl. Bakteriol Parasitenk. Infectionskr. Hyg. Abt. 1: Orig. Reihe B., 175, 363-368.

Marier R., Wells J.C., Swanson R.C., Callahan W., Mehlman I.J., 1973. An outbreak of enteropathogenic Escherichia coli food borne diseases traced to imported French cheese. Lancet, 1367-1378.

Marshall R.T., Adams D.M., Morgan D.R., Olsen N.F., White C.H., 1975. Microbiological methods for dairy products. In : Standard methods for the examination of dairy products, G.H. Richardson Ed., Washington, D.C., 212. 
MarTh E.H., 1969. Salmonellae and salmonellosis associated with milk and milk products. A review. J. Dairy Sci., 52, 283-315.

Massa S., Trovatelli L.D., Vasta E., Barbieri G., Dondi C., 1986. Study of the microbiological and chemical characteristics of Sogliano "pit" cheese. Microbiol. Alim. Nutr., 4, 183-190.

Minor T.E., Marth E.H., 1972. Staphylococcus aureus and staphylococcal food intoxications. A review. III. Staphylococci in dairy foods. J. Milk Food Technol., 35, 77-82.

Ottogalli G., Rondinini G., Germodari I., 1978. Saggi per la valutazione della qualità igienica del formaggio. Atti Convegno SIMA. Dicembre, Milano.

Park H.S., Marth E.H., Olson N.F., 1973. Fate on the enteropathogenic strains of Escherichia coli during the manufacture and ripening of Camembert Cheese. J. Milk Food Technol., 36, 543-546.

Rash K.E., Kosikowski F.V., 1982. Behaviour of enteropathogenic Escherichia coli in Camembert cheese made from ultrafiltred milk. J. Food Sci., 47, 728-732.

Ratnam S., March S., 1986. Laboratory studies on salmonella-contamined cheese involved in major outbreak of gastroenteritis. J. Appl. Bacteriol., 61, 51-56.

Ricci N., Manuppella A.M., Melaragno E., Di Marzo V., Laurelli T., 1984. Intossicazione da Stafilococco in provincia di Isernia. Boll. Epid. Naz., 7, 1-5.

Ritter P., 1975. Problèmes hygiéniques dans la producion du fromage. Ind. Latte, 11, 65-77.

Thrasher S., Richardson G.H., 1980. Comparative study of the stomacher and the waring blender for homogenization of high-fat dairy foods. J. Food Prot., 43, 763-764.

Tiecco G., 1984. Microbiologia degli alimenti di origine animale. III Ed. Edagricole, Bologna. 